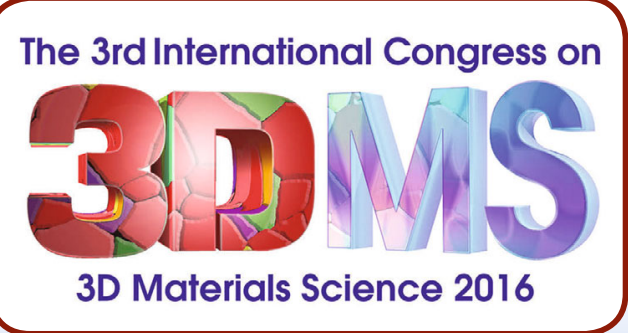

\title{
Assessing Progress on 3D Materials Science Closing Panel Discussion Highlights from 3DMS2016
}

Laura T. Beringer, Ryan Harrison, Will Lenthe, and George Spanos

\section{Background}

The International Congress on 3D Materials Science (3DMS) was established in 2012 as a destination for networking and discussion on the state of the art and future directions in three dimensional (3D) characterization, modeling, and data management/analysis within the materials science and engineering community. The third installment in this conference series-3DMS2016 - took place July 10-13, 2016 in St. Charles, Illinois. As the 3D materials science community moves forward in anticipation of the fourth world congress in this series (3DMS2018), it is important to consider some common themes, challenges, future directions, and recommendations highlighted at 3DMS2016. While not an all-inclusive report of 3DMS2016, this article reviews some of the key themes that were raised at 3DMS2016 during the final panel discussion on critical issues impacting on the future growth of the field.

\section{Theme Identification}

3DMS2016 focused on six key overarching areas of 3D materials science: experimental techniques for 3D data acquisition; image processing and digital representation of 2D and 3D microstructural data; processmicrostructure-property relationships in $3 \mathrm{D}$; advances in reconstruction algorithms; advances in 3D materials modeling; and future directions and challenges for 3D materials sciences. Session chairs captured inputs each day from presentations and discussions as the basis for the closing panel discussion, titled, "Future Directions and Challenges for 3D Materials Science." A number of key themes were identified through this process. For example, there is a rich knowledge base that can be gained from the signal processing and other communities that process various types of data. This provides the materials science and engineering community with an opportunity to take advantage of this knowledge base and apply it to 3DMS techniques in areas of greatest benefit. The need to better link experimental and computational data to predict microstructure, properties, and material behavior was also a prevalent theme, with recognition that this should be a strong focus for future research.

Another major area of discussion was how to best approach and leverage the multiplicity of experimental, modeling, and data management techniques in this field. Issues included optimally combining multiple experiments and models, and creating modular designsversus rigidly integrated systems - for complex computational and experimental systems. Using standard segmentation workflows for experiential techniques was also recognized to be essential for adoption of these techniques by industry. Additional research areas highlighted at 3DMS2016 included using incomplete data to extrapolate to accurate representations of 3D microstructures and property predictions, and/or determining statistically significant material behavior from a limited number of measurements. Being able to run simulations at synchrotron and/or neutron facilities at the same time the experiments are being run also emerged as a prevalent theme.

\section{"Future Directions" Panel Discussion Summary}

To facilitate further dialogue on these and related topics, the conference organizers structured the following leading questions for the closing interactive panel 
discussion, based on the feedback from the session chairs:

1. How far is the 3DMS community from adopting or suggesting standards toward segmentation and data processing requirements? Is image processing and data analysis a viable course addition for materials science students?

2. How do we go about being more efficient with our data collection strategies?

3. How can we do a better job of bringing together theoretical, experimental, and software research efforts?

4. What does the 3D materials science community need to do to move more from model systems to real industrial materials (e.g., additive manufacturing)?

5. What is the modeling community doing to incorporate nanometer scale characterization into analyses?

6. We are learning a great deal as a community from medical imaging protocols as well as the image processing community. Are there other communities from whom the 3DMS community should also learn?

Consideration of the leading questions by both the panel participants and congress attendees gave rise to the following comments and recommendations concerning the future of the $3 \mathrm{D}$ materials science community:

\section{Data Standards and Processing in the 3DMS Community}

An ultimate goal is to establish formalized standards and workflows for as many 3DMS techniques as possible, and potentially for different classes of materials as well. In order to achieve such standards, the community needs to develop and employ quantitative tools that are objective. It is equally important that the community as a whole recognizes and accepts the need to work together towards developing such standards and tools. One approach is to have specific samples measured at different facilities, and then use those results to develop standards and calibration protocols. There are several areas within the 3DMS community where implementing standards should be considered. One example is the area of porosity, where standards for 3D analyses could be developed by academia and government laboratories, working in collaboration with industry. Another example is potential partnering with aerospace companies in the development of standards for micro-texture evaluation in titanium alloys.

\section{D Materials Data Collection and Management Strategies}

Another set of recommendations explored at the panel discussion focused on leveraging examples from other communities on how to collect and manage data. For example, in particle physics research, CERN (the European Organization for Nuclear Research) utilizes a tiered approach toward data storage and reduction. It is especially important with 3D datasets to develop data optimization algorithms to reconstruct the data in a short amount of time (minutes vs. days). Determining which data collection method is appropriate for a given material is challenging. However, when considering mechanical testing and experimentation, utilizing models to estimate the final format and utility of the resultant $3 \mathrm{D}$ data in advance can be helpful in determining which type of experiments will provide the best value.

\section{Bridging the Gap: Theoretical, Experimental, and Software Research}

Recent research in 3D materials science has led to better bridging between computational and experimental efforts. Through collaboration with software engineers, some communities have been able to overcome some of the major hurdles present in bridging not only computational and experimental efforts, but time and length scales as well. For instance, although data format can be a barrier to running a computational model, developing new software and standards can help mitigate this problem. The "Dream3D" tool suite is one such example where 3D materials data can be efficiently implemented, managed, and formatted within a robust software package. Sharing methods within the community on how to create appropriate data formats that interface

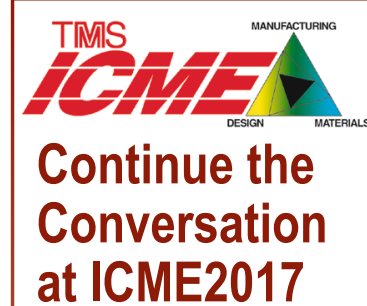

While the 3DMS Congress series offers a unique focus on issues specific to 3D materials science, relevant topics will also be explored at the 4th World Congress on Integrated Computational Materials Engineering (ICME 2017), set for May 21-25, 2017 in Ypsilanti, Michigan.

Visit www.tms.org /ICME2017 to review the technical program and the list of confirmed invited speakers.

Registration includes both a hard copy and free electronic access to the ICME2017 proceedings. 
with software is important, and from a funding perspective, if federal agencies allow funding for this type of work, it could accelerate implementation. Ultimately, working together with software engineers and helping them understand the challenges associated with 3D materials science data, perhaps by sharing discussions and results such as those at 3DMS2016, will enable the field of three-dimensional materials science to move forward more rapidly.

\section{Moving from Model Systems to Real Industrial Materials}

Development of new industry-relevant materials systems based on the results of modeling and/or experimental work on model systems is difficult due to intellectual property (IP) and other restrictions that prevent the public dissemination of some research results. In this regard, scientists and engineers can look for the best opportunities in the precompetitive realm in which they can collaborate and share information. Another suggestion toward moving closer to real industrial materials in 3DMS analyses is to make stronger, more quantitative links between $3 \mathrm{D}$ computational and experimental results. One example is the quantitative incorporation of $3 \mathrm{D}$ characterization dataon microstructural features where cracks initiate - into models of materials behavior and failure. A benchmarking initiative was also discussed, where model materials could be used to develop some standard practices, and analyses could then be translated to industrially relevant materials. This could also involve open publishing of these results so that the field could be advanced at a more rapid pace.

\section{Challenges Associated with Nanoscale 3D Characterization}

Characterization at the nanoscale can present its own unique challenges. Although it has been a goal of the field to enable multiscale modeling which bridges the gap across nano-, micro-, and macroscales, 3D characterization of materials at the nanoscale typically involves a limited range of view, with the potential that critical data from that material that can be lost. Additionally, uncertainly associated with experimental nanoscale 3D data can present difficulties in modeling nanoscale behavior, particularly when those models rely on the experimental data for initial input and/or validation.

\section{Other Communities that Could Provide Useful Guidance to 3DMS}

The panel discussed mechanical engineering as one such example of a field from which the 3DMS community could gain valuable insights. In particular, computer-aided design and analysis, as well as manufacturing related to mechanical engineering could shed light on potential avenues for growth in 3DMS. The computational mechanics community was identified as another group to leverage. Specifically, in both computational fluid mechanics and computational solid mechanics, a serious effort is underway to incorporate error analysis and adaptive computation to enhance model reliability and accuracy. Both of these fields employ formalized methods that have clear applications in 3D materials science. Fields such as mechanical engineering and computational mechanics often view things from a workflow perspective, and this type of thinking needs to be more strongly incorporated into the 3DMS community as well.

\section{Summary and Future Outlook}

The pervasive themes that emerged from 3DMS2016 focused on developing 3D materials data standards and improving communications within the 3D materials science community, as well as with various other scientific communities. Looking forward, many of the highlights and recommendations that were explored during the "Future Directions" panel discussion at 3DMS2016 are expected to be reflected in the technical programming of 3DMS2018, which should demonstrate and review the further evolution of this field.

Laura Beringer is the Technical Specialist and George Spanos is the Technical Director at TMS; Ryan Harrison is a Ph.D. candidate, Carnegie Mellon University, Materials Science and Engineering Department; Will Lenthe is a Ph.D. candidate, University of California at Santa Barbara, Materials Science and Engineering Department.

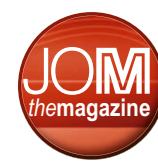

